\title{
Regulation of a minimal transcriptome by repeat domain proteins
}

Nicola Ferreira ${ }^{1}$, Oliver Rackham ${ }^{1}$ and Aleksandra Filipovska ${ }^{1 *}$

${ }^{1}$ Harry Perkins Institute of Medical Research and The University of Western Australia,

School of Chemistry and Biochemistry, Nedlands, 6009, Perth, Western Australia, Australia

*Correspondence: aleksandra.filipovska@uwa.edu.au (A. Filipovska) 


\begin{abstract}
Repeat proteins post-transcriptionally regulate the expression of the mammalian mitochondrial genome at the level of transcription, processing, maturation, and translation. Defects in the regulation of mitochondrial gene expression due to mutations in genes encoding repeat proteins can lead to mitochondrial dysfunction and disease, however the molecular mechanisms that regulate mitochondrial gene expression and how defects in these processes cause disease still remains poorly understood. Recently solved crystal structures, characterisation of the new genetic models, and use of RNA sequencing (RNA-Seq) technologies have greatly expanded our current understanding of mitochondrial repeat proteins and biology.
\end{abstract}

Keywords: Pentatricopeptide repeat protein, mitochondrial transcription termination factor, RNA-binding, mitochondrial RNA, gene expression 


\section{Highlights}

- MTERFs act as multi-functional proteins in regulating post-transcriptional control

- RNA processing links transcription and translation via assembly of the mitoribosome

- $\quad 5^{\prime}$ mt-tRNA end cleavage by RNase P precedes $3^{\prime}$ tRNA end cleavage by RNase Z

- Human MRPP3 is an L-shaped molecule that resides in an auto-inhibitory state

- Impaired RNA processing can predispose to an adult-onset metabolic syndrome 


\section{Regulation of mammalian mitochondrial gene expression by modular proteins}

The mammalian mitochondrial DNA (mtDNA) is compact, circular, double-stranded genome, containing only 37 genes for 2 rRNAs, 22 tRNAs and 11 mRNAs. The mitochondrial mRNAs (mt-mRNAs) encode 13 polypeptides that are subunits of three respiratory complexes (Complex I, III and IV) and the ATP synthase, which together with Complex II form the oxidative phosphorylation (OXPHOS) system required for the majority of energy generation in cells (reviewed in $[1,2]$ ). The expression of mammalian mtDNA is regulated predominantly at a post-transcriptional level by many nuclear encoded RNAbinding proteins that are imported inside mitochondria post-translationally to transcribe, stabilize, modify, translate and degrade mt-RNAs [3]. The known roles of nuclear encoded mitochondrial RNA-binding proteins (mtRBPs) have been reviewed recently $[1,2,4,5]$. Here we discuss the role of two families of mtRBPs that consist of characteristic repetitive protein motifs, mitochondrial transcription termination factors (mTERF) that can bind mtDNA and mtRNA and pentatricopeptide repeat (PPR) domain proteins [6-8].

The MTERF proteins are a group of structurally similar proteins with variably repeated MTERF-motifs that form a half-donut-shape, right-handed superhelix, where the positively charged concave side forms a path for nucleic acid binding [9-12]. In humans there are four human MTERF proteins: MTERF1, MTERF2, MTERF3, and MTERF4, all of which reside inside mitochondria $[6,9,13-17]$. The molecular structures and roles of most mammalian MTERF proteins in different aspects of mitochondrial gene expression have been well characterized, although little is known about the role of MTERF2 in mitochondria besides its affinity for mtDNA binding and association with the nucleoid [6,9,13-18].

PPRs were initially discovered in plant organelles as a large family of up to 800 proteins $[7,19]$ that were only recently identified to bind RNA in a sequence specific and 
modular fashion [20-24]. In mammals there are only seven PPR proteins (Figure 1) that are exclusively mitochondrially localized and regulate mitochondrial gene expression from transcription to protein synthesis: the mitochondrial RNA polymerase (POLRMT) [25], leucine-rich pentatricopeptide repeat cassette (LRPPRC) protein [26-29], mitochondrial RNase P protein 3 (MRPP3) [30-32], pentatricopeptide repeat domain proteins 1, 2, and 3 (PTCD1, PTCD2, and PTCD3) [33-36], and mitochondrial ribosomal protein of the small subunit 27 (MRPS27) [37].

MtDNA is transcribed by POLRMT as two long polycistronic transcripts that span almost the entire length of the heavy or light strand of the genome (Figure 2) [38]. MTERF1 has been identified to bind within the tRNA ${ }^{\text {Leu(UUR) }}$ gene $[9,13,14,39]$ and in vivo studies have shown that its binding blocks transcription to prevent transcriptional interference at the light strand promoter (LSP) [15]. The two polycistronic transcripts are processed according to the tRNA punctuation model (Figure 2), whereby mitochondrial rRNAs (mt-rRNAs) and mitochondrial mRNAs (mt-mRNAs) are interspersed by mitochondrial tRNAs (mt-tRNAs), which act as "punctuation marks" for processing [40,41]. Processing of the polycistronic transcripts involves the excision of mt-tRNAs at their 5' ends by RNase P complex, which in mammals consists of three proteins, MRPP1, MRPP2, and MRPP3 [30-32,34,42], and at their 3' ends by RNase Z, which is ELAC2 [34,43]. PTCD1 associates with ELAC2 to assist in mttRNA processing [34]. However, there are four exceptions to the tRNA punctuation model (Figure 3) which include the $3^{\prime}$ end of $m t-N D 6,5^{\prime}$ end of $m t-C O 1,5^{\prime}$ end of $m t-C Y B$ and between $m t-A T P 6$ and $m t-C O 3$ [40]. It is not entirely clear which other enzymes process these non-canonical sites although PTCD2 has been suggested to be required for processing the $5^{\prime}$ end of the $m t-C Y B$ transcript. Loss of the fas-activated serine/threonine kinase 5 (FASTKD5) results in the accumulation of unprocessed transcripts from the $5^{\prime}$ end of $m t-C Y B$ and the $5^{\prime}$ end of $m t-C O 1$ and between $m t-A T P 8 / 6-C O 3[35,44]$ and disruption of fas- 
activated serine/threonine kinase 4 (FASTKD4) has also been shown to cause the accumulation of the $m t N D 5-C Y B$ precursor transcript. It has been suggested that the FASTKD protein family contain a putative endonuclease in the RNA-binding domain, RAP [45]. However, it still remains to be confirmed if PTCD2 has endonuclease activity or recruits endonucleases that are not yet characterized or identified.

The cleaved RNA transcripts then undergo maturation mediated by the LRPPRC/SLIRP complex $[28,46]$ which includes polyadenylation of mt-mRNAs by the poly(A) polymerase (PAP), modification of tRNAs by a range of enzymes [4] and methylation of rRNAs by a variety of enzymes, including NSUN4 $[6,11,47]$, followed by translation on mitochondrial ribosomes [48-50]. Mitochondrial ribosomes contain two PPR proteins within the small ribosomal subunit (PTCD3 and MRPS27) and are assembled co-transcriptionally [42]. Their assembly is mediated by a number of different factors, some characterized such as DDX28 [51] and MTERF3 [17], with additional players yet to be characterized.

The variation in the levels of mt-RNAs underlies the significance of RNA-binding proteins in the post-transcriptional regulation of mitochondrial gene expression [52]. Repeat domain proteins play key roles in every aspect of mitochondrial gene expression, from transcription to protein synthesis and mutations or loss of these proteins can lead to mitochondrial dysfunction and disease $[6,15,16,26,28,42,53,54]$.Here we review the current advances towards understanding the role of mammalian MTERF and PPR proteins in mitochondrial biology and pathology.

\section{Mitochondrial transcription termination factors (MTERFs)}

\subsection{Mitochondrial transcription termination factor 1 (MTERF1)}

MTERF1 contains 8 MTERF-motifs, comprising of $19 \alpha$-helices [9]. MTERF1 binds in a sequence-specific manner to a 28 bp region within the tRNA ${ }^{\text {Leu(UUR) }}$ gene, downstream of the 
$16 \mathrm{~S}$ mt-rRNA region and was originally thought to promote heavy-strand transcription termination in vitro [13]. However, the role of MTERF1 in heavy-strand transcription termination has since been debated as MTERF1 has been shown to partially terminate transcription from the heavy strand promoter (HSP), while completely terminating transcription from the LSP [14]. The role of MTERF1 in light-stand transcription termination was identified in vivo when both copies of the mouse Mterfl genes were deleted to reveal that MTERF1 is dispensable for rRNA gene transcription regulation and instead it is required, but not essential, for minimizing transcription interference at the light strand promoter [15]. A mechanism of transcription termination by MTERF1 has since been proposed where MTERF1 binds to the major groove of DNA at its target site in the tRNA ${ }^{\text {Leu(UUR) }}$ gene to promote unwinding of the DNA helix and eversion of several key residues to cause base flipping in a stepwise manner, which is critical for transcription termination $[9,55]$. In addition to transcription termination, MTERF1 was proposed to promote rRNA synthesis by binding to both the heavy strand transcription initiation and termination sites to mediate the formation of a DNA loop [39]. A recent report has also implicated MTERF1 in the regulation of mtDNA replication pausing in vitro [56,57], indicating that overall MTERF1 plays an important role in regulating mitochondrial transcription and mtDNA replication.

MTERF1 binds DNA in a site and cell specific manner [9,52], with conserved residues responsible for the sequence-specific binding [9]. Pathogenic mutations in these residues in MTERF1 have been proposed to interfere with MTERF1 binding and transcriptional termination $[9,58]$. Furthermore, MTERF1 has also been shown to have a tissue-specific transcriptional and translational compensatory role in response to decreased mtDNA copy number in a MPV17 knockout mouse model that causes severe mitochondrial DNA depletion [59]. This implicates MTERF1 in diseases caused by impaired mitochondrial gene regulation, and further studies will shed light on its tissue-specific role in disease. 


\subsection{Mitochondrial transcription termination factor 3 (MTERF3)}

MTERF3 is a highly conserved protein that contains 7 MTERF-motifs, consisting of $22 \alpha-$ helices $[10,60]$. MTERF3 has a concave side with a strong positive charge that represents a nucleic-acid binding site, important for its role in binding mtDNA to regulate posttranscriptional control [10]. MTERF3 is essential for life and heart-specific knockout of mouse Mterf3 gene causes respiratory chain defects and consequently mitochondrial dysfunction which results in a shortened life-span [16]. MTERF3 has been shown to associate with the mtDNA light and heavy strand promoter regions, resulting in increased transcription initiation from the LSP and HSP and increased steady-state levels of most mtRNAs. Furthermore MTERF3 was identified to bind the 16S mt-rRNA and to be important in the assembly and maturation of the large mitoribosomal subunit that is essential for assembly of the 55S mitochondrial ribosome [17]. Both studies have implicated the importance of MTERF3 in the regulation of mitochondrial transcription and ribosome biogenesis in vivo.

Recently, the loss of MTERF3 was investigated in cardiomyopathy to show that despite severe OXPHOS defects there were no signs of oxidative stress or production of reactive oxygen species (ROS) [61]. This is particularly important since many studies of heart disease implicate ROS and oxidative stress as the major contributor to cardiomyopathy and cardiac hypertrophy, identifying that there is a complex interplay between mitochondrial dysfunction and subsequent cellular signalling networks that precede heart disease. Mouse models of heart disease where nuclear genes encoding mitochondrial proteins have been knocked out or mutated, are ideal systems to investigate the role of mitochondrial dysfunction in different forms of heart disease.

\subsection{Mitochondrial transcription termination factor 4 (MTERF4)}


MTERF4 is essential for embryo development and heart-specific loss of mouse Mterf4 causes mitochondrial cardiomyopathy, respiratory chain deficiency and shortened life-span [6]. MTERF4 regulates mitochondrial ribosome biogenesis and thereby translation through its association with the ribosomal RNA methyltransferase (NSUN4) $[6,47]$. The binding of MTERF4 to mt-rRNAs occurs via a heterodimer complex with NSUN4, which assists in targeting this protein to the large subunit [6]. The atomic structure of the MTERF4-NSUN4 complex shows that MTERF4 contains 6 MTERF-motifs comprising $18 \alpha$-helices. The molecular structure of this complex shows that NSUN4, which lacks the ability to bind RNA, binds to the C-terminal domain of MTERF4 via specific conserved interactions which enables RNA binding [11,12]. MTERF4 is further required to stimulate the activity of NSUN4 to specifically methylate $16 \mathrm{~S}$ rRNA $[12,47]$. Overall MTERF4 is essential for regulating mitochondrial ribosome biogenesis and translation in vivo.

\section{Pentatricopeptide repeat domain proteins (PPRs)}

\subsection{Mitochondrial RNA polymerase (POLRMT)}

POLRMT is a single-subunit DNA-dependent RNA polymerase that is distantly related to the bacteriophage T7 RNA polymerase $[25,62]$. It is the only mitochondrial RNA polymerase and forms the core component of the transcription machinery along with mitochondrial transcription factor B2 (TFB2M) and mitochondrial transcription factor A (TFAM), which are required by POLRMT to initiate transcription of mitochondriallyencoded genes $[25,63-66]$. The formation of the transcription machinery begins when TFAM binds to the DNA promoter and recruits POLRMT via a protein-protein interaction to ensure promoter-specific transcription [64]. TFB2M then binds to form a transcription initiation complex with POLRMT and TFAM, and together TFAM and TFB2M melt the promoter to initiate transcription by POLRMT 
[64,67-69]. POLRMT also synthesises the RNA primers required for the replication of the mtDNA which often takes precedence over transcription $[54,63]$. POLRMT is essential for survival since its loss causes embryonic lethality and heart- and skeletal muscle-specific knockout of Polrmt in mice leads to premature death as a result of a profound cardiomyopathy by 6 weeks of age [54]. POLRMT is exclusively a mitochondrial protein that does not support the transcription of nuclear encoded genes [66].

POLRMT interacts with the transcription elongation factor of mitochondria (TEFM), and together they can regulate the switch between replication primer formation and gene expression to mediate mtDNA transcription and replication [54,70-72]. TEFM activates and enhances mitochondrial transcription by stimulating the formation of longer transcripts and stabilising the interaction of POLRMT with DNA [71]. POLRMT has been shown to associate with the mitochondrial ribosome (mitoribosome) protein of the large subunit 12 (MRPL12), when this protein is not part of the mitoribosome; this interaction is thought to stabilise POLRMT and influence mitochondrial transcription [73-75]. Further studies have also found POLRMT associated in a small subunit mitochondrial ribosome complex with TFB1M [76], which is a $12 \mathrm{~S}$ ribosomal RNA methyltransferase required for the maturation and assembly of the small subunit of the mitochondrial ribosome [77]. This association is thought to occur independently of transcription and to be distinct from the POLRMT and TFB2M complex to increase the 12S mt-rRNA methyltransferase activity of TFB1M [76]. The atomic structure of the human POLRMT revealed a catalytic carboxy-terminal domain similar to that of phage polymerases and a unique extended $\mathrm{N}$-terminal region containing two PPRs with an AT-rich recognition loop [25]. Functional analysis showed that the PPR domains play no role in transcription initiation, which is carried out by the Cterminal domain. Instead, the crystal structure of the human POLRMT elongation complex shows newly synthesised RNAs exiting towards the PPR domain, suggesting a role for the 
PPR domain in the elongation stage of transcription [78]. Elucidation of the POLRMT termination complex structure may be important in establishing if there is an additional role of the PPRs in POLRMT function.

\subsection{Mitochondrial RNase P protein 3 (MRPP3)}

MRPP3 is a mitochondrial protein containing five PPRs and a metallonuclease domain $[30,31,42]$. Initially, MRPP3 was co-purified with two additional proteins, MRPP1 and MRPP2, which together were identified to form the mitochondrial RNase P complex that processes the 5' ends of mt-tRNAs [32]. MRPP1 and MRPP2 form a stable subcomplex within the RNase P complex, however they are bifunctional proteins with additional cellular functions. MRPP1 belongs to the TRMT10 family and is a $\mathrm{m}^{1} \mathrm{G}_{9}$ methyltransferase responsible for methylating the ninth position on mt-tRNAs [34,79] and may have a role in mt-tRNA binding required for the RNase P complex [80]. MRPP2 is a member of the shortchain dehydrogenase/reductase family and has a $\mathrm{NAD}^{+}$binding domain, but no RNA-binding domains, and may be required for RNase P activity [81] or act as a structural scaffold for the RNase P complex. Mutations in MRPP2 cause mitochondrial disease as a result of impaired RNA processing, providing evidence that it is required for RNase $\mathrm{P}$ activity [82,83]. Until recently, the human mitochondrial RNase $\mathrm{P}$ complex was thought to be unique as a protein only complex that lacks the catalytic RNA component which is generally found in previously characterized nuclear RNase P enzyme complexes [32,84,85]. However, protein-only RNase P (PRORP) enzymes have since been identified to process precursor tRNAs in organelles and nuclei of diverse eukaryotic species [86-89].

The atomic structure of PRORP1, which is a homolog of MRPP3, from Arabidopsis thaliana is V-shaped with a metallonuclease domain linked to a PPR domain by a zincbinding domain [90]. The metallonuclease domain is the active site and is responsible for the 
catalytic activity of the protein, without the need for additional proteins or a catalytic RNA. This active site contains $\mathrm{Mg}^{2+}$ metal ions bound to conserved aspartate residues that are essential for its catalytic activity. The PPR domain is thought likely to provide specificity through its interaction with pre-tRNAs [90]. In contrast to the plant PRORP1, the atomic structure of the human MRPP3 protein has been shown to have a more relaxed, L-shape that is also composed of a PPR domain connected by a central domain containing a highly conserved zinc-binding site bound to a metallonuclease domain [30,31]. Unlike PRORP1, MRPP3 has a distorted and non-productive active site, as metals ions that are necessary for its activity are absent from the active site and the protein exists in an auto-inhibited state [30,31]. Since the human MRPP3 protein requires MRPP1 and MRPP2 for its catalytic activity, it is thought that only upon their binding along with a pre-tRNA substrate that a conformational change is induced to stimulate enzymatic activity [30,31]. Why this additional layer of specificity might be required in mammalian mitochondria is not known. The RNase P complex has been associated with mitochondrial RNA granules that are viewed as centers for RNA processing, maturation, and mitochondrial ribosome biogenesis. These processes occur co-transcriptionally [42] and in close proximity to mitochondrial nucleoids $[44,91-93]$

Knockdown of MRPP3 in HeLa cells has been shown to cause a processing defect with the accumulation of precursor transcripts and a reduced abundance of mature mt-RNAs $[32,34,94]$. However the essential role of MRPP3 was revealed recently where a knockout of Mrpp3 in mice was shown to be embryonic lethal and heart and skeletal-muscle specific knockout of Mrpp3 caused a cardiomyopathy and premature death at 12 weeks of age [42]. Loss of MRPP3 caused a profound processing defect and the accumulation of unprocessed transcripts and the loss of mature mt-RNAs [42]. The loss of mature mt-RNAs was associated with a large increase in transcription driven by increased POLRMT levels that were not 
sufficient to overcome the processing defects. The role of MRPP3 in 3' end cleavage of mttRNAs has been investigated across the entire mitochondrial transcriptome using diverse RNA sequencing (RNA-Seq) techniques in both in vitro and in vivo models where MRPP3 has been knocked down or lost $[34,42]$. Transcriptome wide parallel analyses of RNA ends (PARE) has identified the exact in vivo cleavage sites of RNase P and has demonstrated that cleavage of the $5^{\prime}$ mt-tRNA precedes $3^{\prime}$ mt-tRNA end processing [42]. Recently we have used the same model to elucidate the hierarchy of mitochondrial RNA processing via RNA circularization prior to library preparation and RNA-Seq. Our findings revealed the identities of processing intermediates, their abundance in vivo and showed that RNA processing and degradation can occur simultaneously [95].

The loss of MRPP3 and functional processing of mt-RNAs causes impaired translation of all mitochondrially encoded polypeptides that has downstream consequences on the biogenesis and activity of the mitochondrial respiratory complexes causing general OXPHOS defects and mitochondrial dysfunction [42]. Characterisation of a MRPP3 heart and skeletal muscle-specific knockout mouse model has provided the first evidence of a mechanistic link coupling mitochondrial transcription and translation via the assembly of the mitoribosome [42]. The assembly of the small subunit is impaired in the absence of a mature $12 \mathrm{~S}$ mt-rRNA, while the large subunit mitoribosome proteins form a sub-complex on the precursor $16 \mathrm{~S}$ mt-rRNA, showing that mitoribosomal biogenesis occurs co-transcriptionally [42]. Future studies will be required to understand mitoribosomal assembly in detail and this new model will assist in providing insight into the early assembly of subcomplexes of the mitoribosome.

\subsection{Leucine-rich pentatricopeptide repeat cassette (LRPPRC) protein}


LRPPRC is a mitochondrial protein predicted to contain 30-33 PPR domains and is essential for life $[28,29,96-98]$. LRPPRC was the first human PPR protein to be identified when a $\mathrm{C}$ to T transition mutation at $1119 \mathrm{bp}$ of the LRPPRC open reading frame was shown to change a conserved alanine residue to valine at position 354 causing the rare autosomal recessive French-Canadian variant of Leigh Syndrome (LSFC found in the Saguenay-Lac-Saint-Jean region of Quebec) [26]. Patients with mutations in LRPPRC LSFC have cytochrome $c$ oxidase (COX) deficiency and show clinically distinct symptoms from classical Leigh Syndrome, where the brain and liver are mostly affected and display early-onset neurodegeneration (Leigh disease), liver steatosis, and chronic lactic acidosis $[99,100]$. However, it has been suggested that these symptoms can be affected by other genetic and environmental factors [101]. Recently there have been reports of new LRPPRC mutations that resemble LSFC outside of the French-Canadian population [102]. LSFC patient fibroblasts containing the A354V (Ala ${ }^{354} \rightarrow$ Val) have reduced levels of LRPPRC, impaired translation of mitochondrially-encoded proteins and consequently defects in OXPHOS [27]. Recently it was shown that loss of LRPPRC and SLIRP results in tissue-specific OXPHOS deficiencies in LSFC patients [103].

In vivo investigations have shown that when Lrpprc is deleted in mice it results in embryonic lethality and heart and skeletal muscle-specific loss causes cardiomyopathy and premature death [28]. Loss of LRPPRC results in uncoordinated protein synthesis compromising OXPHOS biogenesis and function [104], similarly to the defects identified in liver-specific deletion of Lrpprc [105]. Lrpprc heart deficient mice also have a significantly reduced abundance of mt-mRNAs, except $m t-N D 6$, which further corroborates with in vitro studies, but also shows increased abundance of mt-rRNAs [28]. Additional studies using heterozygous $\mathrm{Lrpprc}^{+/}$mice or LRPPRC knockdown in cells have identified accumulation of 
some precursor mt-mRNAs $[53,106]$, suggesting that LRPPRC loss may contribute to accumulation of processing intermediates.

LRPPRC binds the Stem-Loop Interacting RNA Binding Protein (SLIRP) in a codependent manner to form a hetero-dimeric protein complex $[27,28,97]$. The PPR domain of LRPPRC and the RNA recognition motif (RRM) domain of SLIRP were recently shown to participate in protein-protein interactions enabling the formation of a stable complex between these two proteins [97]. LRPPRC and the LRPPRC/SLIRP complex, but not SLIRP alone, bind mt-RNA [27,97]. Recently we carried out high throughput RNase footprinting and photoactivatable ribonucleoside-enhanced crosslinking and immunoprecipitation (PARCLIP) to identify the in vivo binding targets of LRPPRC and its role as a mt-RNA chaperone required to relax secondary RNA structures thereby facilitating polyadenylation, RNA stability and coordinated translation [107].

The LRPPRC/SLIRP complex maintains a pool of non-translated mt-mRNAs and maintains mt-mRNA polyadenylation of all mt-mRNAs, except $m t-N D 6$, which is the only non-polyadenylated mt-mRNA $[28,106,108]$. LRPPRC and the LRPPRC/SLIRP complex promote polyadenylation by mitochondrial poly(A) polymerase (mtPAP) to maintain polyadenylated mt-mRNAs, while SLIRP stabilizes LRPPRC by protecting it from degradation $[46,97,108,109]$. The LRPPRC/SLIRP complex specifically stabilizes mtmRNAs with longer half-lives including $m t-C O 1, m t-C O 2$, and $m t-C O 3$ in HeLa cells by possibly suppressing $3^{\prime}$ exonuclease activity mediated by polynucleotide phosphorylase (PNPase) and SUV3 helicase, however the exact mechanism by which this occurs is yet to be described [108]. Although its stable binding partner SLIRP is not essential for survival, its presence is required for the recruitment of mt-mRNAs to the mitochondrial ribosome and controlling the rate of translation [46]. Therefore the LRPPRC/SLIRP is essential for mRNAs stability, polyadenylation and the coordination of mitochondrial protein synthesis. 


\subsection{Pentatricopeptide repeat domain protein 1 (PTCD1)}

PTCD1 contains fifteen PPR domains with no other function domains and it is essential for life $[33,110]$. PTCD1 in cells has been found associated with the leucine mt-tRNAs (mttRNA $^{\text {Leu(UUR) }}$ and tRNA $\left.{ }^{\text {Leu(CUN) }}\right)$ and precursor RNAs containing the leucine mt-tRNAs and affects OXPHOS function $[33,80,94]$. RNA-Seq analyses have indicated that decreased levels of PTCD1 are associated with changes in $3^{\prime}$ end processing of mt-tRNAs, suggesting that PTCD1 may affect RNA processing [34]. Furthermore, low levels of PTCD1 have been found to associate with ELAC2 suggesting that these proteins are in a common complex and through their association they may affect RNA metabolism or co-location within the RNA granules of mitochondria. Our group is investigating the exact molecular role of PTCD1 in vivo currently to identify its role in mitochondrial gene expression.

Recently, we have identified that heterozygous $P t c d 1^{+/}$mice that are haploinsufficient for PTCD1 develop age-induced obesity, glucose intolerance and insulin resistance, hepatic steatosis and cardiac hypertrophy [110]. These pathologies result from perturbed fatty acid metabolism and alterations in metabolic hormones, growth factors, and pro-inflammatory cytokines, which result in downstream tissue-specific changes in the mTOR signalling. The reduction of PTCD1 results in impaired RNA metabolism and decreased protein synthesis. These defects affect the biogenesis of the respiratory complexes and reduce OXPHOS activity, which likely contributes to the liver pathology observed with age [110]. This model demonstrates for the first time that haploinsufficiency of a mitochondrial RNA-binding protein that can cause mild mitochondrial dysfunction that can predispose to an adult-onset metabolic perturbations such as glucose intolerance.

\subsection{Pentatricopeptide repeat domain protein 2 (PTCD2)}


PTCD2 contains at least five PPR domains $[35,96]$ and its role has been investigated in mice homozygous for a Ptcd2 disruption causing truncation of the PTCD2 protein and loss of the full-length protein in all tissues [35]. These mice have decreased Complex III activity, which is predominantly isolated to the heart, although it will be interesting to identify if pathologies in different tissues are observed with age in these mice. Loss of full-length PTCD2 causes a significant accumulation of unprocessed $m t-N D 5-C Y B$ transcripts and consequently reduced levels of mature $m t-N D 5$ and $m t-C Y B$ transcripts [35]. Although PTCD2 does not contain an endonuclease domain, these findings have suggested a role for PTCD2 in RNA processing of the $m t-C Y B$ mRNA, where the $5^{\prime}$ end of the $m t$-CYB is one of the four non-canonical processing sites previously described. PTCD2 has also been implicated in the processing of long non-coding RNA (lncRNA) as knockdown of PTCD2 in HeLa cells causes reduction of $m t-N D 5$ lncRNA and the $m t-N D 6$ lncRNA that forms the $3^{\prime}$ UTR of the $m t-N D 5$ mRNA

[111]. The exact mechanisms by which PTCD2 affects the processing of the non-canonical site of the $5^{\prime}$ end of the $m t-C Y B$ mt-mRNA and $m t-N D 5$ and $m t-N D 6$ lcRNA and the role of lncRNAs in mitochondria remain to be elucidated.

\subsection{Pentatricopeptide repeat domain protein 3 (PTCD3)}

During evolution, the mammalian mitochondrial ribosome has diverged from its bacterial predecessor with a reduction in RNA content and acquisition of additional mitochondrialspecific proteins. These unique mitoribosomal proteins are predicted to have important and specific roles in the recognition of mRNAs and mitochondrial translation (reviewed in [48]). PTCD3, also known as MRPS39, is one of these mammalian-specific supernumerary proteins and is also a member of the PPR family as it contains fifteen PPRs [36,96]. Knockdown of PTCD3 in cells does not affect RNA metabolism, but severely disrupts protein synthesis and causes global OXPHOS defects [36]. PTCD3 associates with the 12S rRNA and co- 
immunoprecipitates with mitochondrial ribosomal proteins of the small subunit [36], acting as a component of the small subunit of the mitoribosome [36,112]. In addition, PTCD3 has been found associated with the mitochondrial translation initiation factor 3 (mtIF3) via the small subunit, which facilitates the dissociation of the large subunit and formation of an initiation complex to initiate mitochondrial translation [113]. In a separate study PTCD3 was found associated with immature colon carcinoma transcript 1 protein (ICT1), which is a mitochondrial ribosomal protein of the large subunit 58 (MRPL58) and has a role as a translation release factor [114], suggesting that PTCD3 associates with translation factors through the assembled mitoribosome. PTCD3 also associates with the mitochondrial ribosomal proteins MRPS32 and MRPS29, which are also supernumerary proteins [112,114116]. The cryoEM structure of the bovine and human mitoribosomes validated PTCD3 as the largest protein component of the small ribosomal subunit [49,50,117], positioned at the head of the subunit and in close proximity to the entrance of the mRNA channel $[49,50,112,117]$. Based on its location PTCD3 could potentially guide mt-mRNAs into the ribosomal mRNA channel at the entry site, however its exact role in translation is currently under investigation. PTCD3 has also been found to associate with the mitochondrial transcription elongation factor (TEFM), mitochondrial RNA polymerase (POLRMT), and a putative dead-box RNA helicase (DHX30) [70], suggesting a potential role of PTCD3 in transcription or that their association is a consequence of the close proximity of the mitoribosome to the transcription machinery, as these systems function interdependently.

\subsection{Mitochondrial ribosomal protein of the small subunit 27 (MRPS27)}

MRPS27 is another supernumerary ribosomal protein of the small subunit and member of the PPR family as it contains six PPR domains [118]. The MRPS27 gene is positioned adjacent to the PTCD2 gene and shares $17.8 \%$ identity with PTCD2, however despite their sequence 
similarity, these proteins are functionally very different [35]. MRPS27 associates with the $12 \mathrm{~S}$ mt-rRNA and is required for mitochondrial protein synthesis and knockdown of MRPS27 in cells causes general OXPHOS decline [37]. The cryoEM structure of the mammalian mitoribosome localized MRSP27 at the bottom of the body of the mitochondrial small subunit suggesting its role in forming a newly discovered protein-RNA inter-subunit bridge that connects the large and small subunit $[49,50,117]$. Biochemical studies have found MRPS27 associated with MRPL58 (ICT1) [114], MRPS29 (DAP3) [119], mitochondrial ribosome assembly factor Era G-protein-like 1 (ERAL1) [120], and the human homolog of a plant NO-associated protein, hNOA1 [121]. The role of these interactions and the exact function of MRPS27 in mitochondrial translation is currently under investigation.

\section{Concluding remarks}

Each of the seven mammalian PPR and four MTERF proteins have diverse roles in the posttranscriptional control of mitochondrial gene expression. MTERF3, MTERF4, LRPPRC, POLRMT, MRPP3 and PTCD1 are essential for life and LRPPRC has been implicated in human disease, which illustrates the importance of gene expression for mitochondrial biogenesis and function. Significant progress has been made into unravelling key aspects of mitochondrial biology and disease, to improve our understanding of post-transcriptional control of gene expression. However, there are many gaps in our knowledge of repeat proteins in biology and disease. Filling these gaps will be critical in establishing the exact roles and mechanisms of repeat proteins in controlling gene expression, which can provide insights into pathogenesis of diseases and new avenues for drug development, as well as fundamental insight into the regulation of the minimal mammalian genome. The continual advances in technologies such as next generation sequencing and the generation of new genetic models are the next frontier for the mitochondrial gene expression field. 


\section{Acknowledgements}

Work in our laboratories was supported by fellowships and project grants from the National Health and Medical Research Council (APP1058442, APP1045677, APP1041582, APP1023460, APP1005030, APP1043978 to AF and OR), Australian Research Council (to AF and OR), the Cancer Council of Western Australia (to OR), and the Alexander von Humboldt Foundation (to AF and OR). NF is supported by a University of Western Australia Top-Up Scholarship and Australian Postgraduate Award.

Figure 1: Schematic representation of the seven mammalian PPR proteins and four mTERF proteins. Repeat proteins were identified using TPRpred [122], sequence inspection, or by examination of the crystal structures, in the case of MRPP3 and POLRMT [25,30,31] and MTERF repeats were identified in the crystal structures of MTERF1 [9-12] or by sequence comparisons.

Figure 2: Schematic of the human mitochondrial genome and illustration of the mammalian tRNA punctuation model

Figure 3: Schematic of the four exceptions to the mammalian tRNA punctuation model

\section{References}

[1] O. Rackham, T.R. Mercer, A. Filipovska, The human mitochondrial transcriptome and the RNA-binding proteins that regulate its expression, Wiley Interdisciplinary Reviews: RNA. 3 (2012) 675-695. doi:10.1002/wrna.1128.

[2] B.M. Hällberg, N.-G. Larsson, Making proteins in the powerhouse, Cell Metab. 20 (2014) 226-240. doi:10.1016/j.cmet.2014.07.001.

[3] I.D. Small, O. Rackham, A. Filipovska, Organelle transcriptomes: products of a deconstructed genome, Curr. Opin. Microbiol. 16 (2013) 652-658. 
[4] T. Suzuki, A. Nagao, T. Suzuki, Human Mitochondrial tRNAs: Biogenesis, Function, Structural Aspects, and Diseases, Annu. Rev. Genet. 45 (2011) 299329. doi:10.1146/annurev-genet-110410-132531.

[5] J. Rorbach, M. Minczuk, The post-transcriptional life of mammalian mitochondrial RNA, Biochem. J. 444 (2012) 357-373. doi:10.1042/BJ20112208.

[6] Y. Cámara, J. Asin-Cayuela, C.B. Park, M.D. Metodiev, Y. Shi, B. Ruzzenente, et al., MTERF4 Regulates Translation by Targeting the Methyltransferase NSUN4 to the Mammalian Mitochondrial Ribosome, Cell Metab. 13 (2011) 527-539. doi:10.1016/j.cmet.2011.04.002.

[7] I. Small, The PPR motif - a TPR-related motif prevalent in plant organellar proteins, Trends Biochem. Sci. 25 (2000) 45-47. doi:10.1016/S09680004(99)01520-0.

[8] C. Schmitz-Linneweber, I. Small, Pentatricopeptide repeat proteins: a socket set for organelle gene expression, Trends Plant Sci. 13 (2008) 663-670. doi:10.1016/j.tplants.2008.10.001.

[9] E. Yakubovskaya, E. Mejia, J. Byrnes, E. Hambardjieva, M. Garcia-Diaz, Helix Unwinding and Base Flipping Enable Human MTERF1 to Terminate Mitochondrial Transcription, Cell. 141 (2010) 982-993.

doi:10.1016/j.cell.2010.05.018.

[10] H. Spåhr, T. Samuelsson, B.M. Hällberg, C.M. Gustafsson, Structure of mitochondrial transcription termination factor 3 reveals a novel nucleic acidbinding domain, Biochem. Biophys. Res. Commun. 397 (2010) 386-390. doi:10.1016/j.bbrc.2010.04.130.

[11] H. Spåhr, B. Habermann, C.M. Gustafsson, N.-G. Larsson, B.M. Hallberg, Structure of the human MTERF4-NSUN4 protein complex that regulates mitochondrial ribosome biogenesis, PNAS. 109 (2012) 15253-15258. doi:10.1073/pnas.1210688109/-/DCSupplemental.

[12] E. Yakubovskaya, K.E. Guja, E. Mejia, S. Castano, E. Hambardjieva, W.S. Choi, et al., Structure of the Essential MTERF4:NSUN4 Protein Complex Reveals How an MTERF Protein Collaborates to Facilitate rRNA Modification, Struct./Fold. Des. 20 (2012) 1940-1947. doi:10.1016/j.str.2012.08.027.

[13] B. Kruse, N. Narasimhan, G. Attardi, Termination of transcription in human mitochondria: Identification and purification of a DNA binding protein factor that promotes termination, Cell. 58 (1989) 391-397. doi:doi.org/10.1016/00928674(89)90853-2.

[14] J. Asin-Cayuela, M. Helm, G. Attardi, A Monomer-to-Trimer Transition of the Human Mitochondrial Transcription Termination Factor (mTERF) Is Associated with a Loss of in Vitro Activity, J. Biol. Chem. 279 (2004) 15670-15677. doi:10.1074/jbc.M312537200.

[15] M. Terzioglu, B. Ruzzenente, J. Harmel, A. Mourier, E. Jemt, M.D. López, et al., MTERF1 Binds mtDNA to Prevent Transcriptional Interference at the LightStrand Promoter but Is Dispensable for rRNA Gene Transcription Regulation, Cell Metab. 17 (2013) 618-626. doi:10.1016/j.cmet.2013.03.006.

[16] C.B. Park, J. Asin-Cayuela, Y. Cámara, Y. Shi, M. Pellegrini, M. Gaspari, et al., MTERF3 Is a Negative Regulator of Mammalian mtDNA Transcription, Cell. 130 (2007) 273-285. doi:10.1016/j.cell.2007.05.046.

[17] A. Wredenberg, M. Lagouge, A. Bratic, M.D. Metodiev, H. Spåhr, A. Mourier, et al., MTERF3 Regulates Mitochondrial Ribosome Biogenesis in Invertebrates and Mammals, PLoS Genet. 9 (2013) e1003178-16. 
doi:10.1371/journal.pgen.1003178.

[18] M. Pellegrini, J. Asin-Cayuela, H. Erdjument-Bromage, P. Tempst, N.-G. Larsson, C.M. Gustafsson, MTERF2 is a nucleoid component in mammalian mitochondria, Biochim. Biophys. Acta. Bioenerg. 1787 (2009) 296-302. doi:10.1016/j.bbabio.2009.01.018.

[19] S. Aubourg, N. Boudet, M. Kreis, A. Lecharny, In Arabidopsis thaliana, 1\% of the genome codes for a novel protein family unique to plants, Plant Mol. Biol. 42 (2000) 603-613. doi:10.1023/A:1006352315928.

[20] E. Delannoy, W.A. Stanley, C.S. Bond, I.D. Small, Pentatricopeptide repeat (PPR) proteins as sequence-specificity factors in post-transcriptional processes in organelles, Biochem. Soc. Trans. 35 (2007) 1643-1647.

doi:10.1042/BST0351643.

[21] A. Barkan, M. Rojas, S. Fujii, A. Yap, Y.S. Chong, C.S. Bond, et al., A Combinatorial Amino Acid Code for RNA Recognition by Pentatricopeptide Repeat Proteins, PLoS Genet. 8 (2012) e1002910-8. doi:10.1371/journal.pgen.1002910.

[22] Y. Yagi, S. Hayashi, K. Kobayashi, T. Hirayama, T. Nakamura, Elucidation of the RNA Recognition Code for Pentatricopeptide Repeat Proteins Involved in Organelle RNA Editing in Plants, PLoS ONE. 8 (2013) e57286-8. doi:10.1371/journal.pone.0057286.

[23] M. Takenaka, A. Zehrmann, A. Brennicke, K. Graichen, Improved Computational Target Site Prediction for Pentatricopeptide Repeat RNA Editing Factors, PLoS ONE. 8 (2013) e65343-12. doi:10.1371/journal.pone.0065343.

[24] S. Coquille, A. Filipovska, T. Chia, L. Rajappa, J.P. Lingford, M.F.M. Razif, et al., An artificial PPR scaffold for programmable RNA recognition, Nature Comm. 5 (2014) 1-9. doi:10.1038/ncomms6729.

[25] R. Ringel, M. Sologub, Y.I. Morozov, D. Litonin, P. Cramer, D. Temiakov, Structure of human mitochondrial RNA polymerase, Nature. 478 (2011) 269273. doi:10.1038/nature10435.

[26] V.K. Mootha, P. Lepage, K. Miller, J. Bunkenborg, M. Reich, M. Hjerrild, et al., Identification of a gene causing human cytochrome c oxidase deficiency by integrative genomics, Proc. Natl. Acad. Sci. USA. 100 (2003) 605-610.

[27] F. Sasarman, C. Brunel-Guitton, H. Antonicka, T. Wai, E.A. Shoubridge, LRPPRC and SLIRP Interact in a Ribonucleoprotein Complex That Regulates Posttranscriptional Gene Expression in Mitochondria, Mol. Biol. Cell. 21 (2010) 1315-1323. doi:10.1091/mbc.E10.

[28] B. Ruzzenente, M.D. Metodiev, A. Wredenberg, A. Bratic, C.B. Park, Y.C.A. mara, et al., LRPPRC is necessary for polyadenylation and coordination of translation of mitochondrial mRNAs, EMBO J. 31 (2012) 443-456. doi:10.1038/emboj.2011.392.

[29] S. Mili, S. Pinol-Roma, LRP130, a Pentatricopeptide Motif Protein with a Noncanonical RNA-Binding Domain, Is Bound In Vivo to Mitochondrial and Nuclear RNAs, Mol. Cell. Biol. 23 (2003) 4972-4982. doi:10.1128/MCB.23.14.4972-4982.2003.

[30] F. Li, X. Liu, W. Zhou, X. Yang, Y. Shen, Auto-inhibitory Mechanism of the Human Mitochondrial RNase P Protein Complex, Sci Rep. 5 (2015) 9878-7. doi:10.1038/srep09878.

[31] L. Reinhard, S. Sridhara, B.M. Hällberg, Structure of the nuclease subunit of human mitochondrial RNase P, Nucleic Acids Res. 43 (2015) 5664-5672. doi:10.1093/nar/gkv481. 
[32] J. Holzmann, P. Frank, E. Loffler, K.L. Bennett, C. Gerner, W. Rossmanith, RNase P without RNA: Identification and Functional Reconstitution of the Human Mitochondrial tRNA Processing Enzyme, Cell. 135 (2008) 462-474. doi:10.1016/j.cell.2008.09.013.

[33] O. Rackham, S.M.K. Davies, A.M.J. Shearwood, K.L. Hamilton, J. Whelan, A. Filipovska, Pentatricopeptide repeat domain protein 1 lowers the levels of mitochondrial leucine tRNAs in cells, Nucleic Acids Res. 37 (2009) 5859-5867. doi:10.1093/nar/gkp627.

[34] M.I.G. Lopez Sanchez, T.R. Mercer, S.M.K. Davies, A.-M.J. Shearwood, K.K.A. Nygård, T.R. Richman, et al., RNA processing in human mitochondria, Cell Cycle. 10 (2011) 2904-2916. doi:10.4161/cc.10.17.17060.

[35] F. Xu, C. Ackerley, M.C. Maj, J.B.L. Addis, V. Levandovskiy, J. Lee, et al., Disruption of a mitochondrial RNA-binding protein gene results in decreased cytochrome $\mathrm{b}$ expression and a marked reduction in ubiquinol-cytochrome $\mathrm{c}$ reductase activity in mouse heart mitochondria, Biochem. J. 416 (2008) 15-26. doi:10.1042/BJ20080847.

[36] S.M.K. Davies, O. Rackham, A.-M.J. Shearwood, K.L. Hamilton, R. Narsai, J. Whelan, et al., Pentatricopeptide repeat domain protein 3 associates with the mitochondrial small ribosomal subunit and regulates translation, FEBS Lett. 583 (2009) 1853-1858. doi:10.1016/j.febslet.2009.04.048.

[37] S.M.K. Davies, M.I.G.L. Sanchez, R. Narsai, A.-M.J. Shearwood, M.F.M. Razif, I.D. Small, et al., MRPS27 is a pentatricopeptide repeat domain protein required for the translation of mitochondrially encoded proteins, FEBS Lett. 586 (2012) 3555-3561. doi:10.1016/j.febslet.2012.07.043.

[38] C.M. Gustafsson, M. Falkenberg, N.-G. Larsson, Maintenance and Expression of Mammalian Mitochondrial DNA, Annu. Rev. Biochem. 85 (2016) 133-160. doi:10.1146/annurev-biochem-060815-014402.

[39] M. Martin, J. Cho, A.J. Cesare, J.D. Griffith, G. Attardi, Termination FactorMediated DNA Loop between Termination and Initiation Sites Drives Mitochondrial rRNA Synthesis, Cell. 123 (2005) 1227-1240. doi:10.1016/j.cell.2005.09.040.

[40] D. Ojala, J. Montoya, G. Attardi, tRNA punctuation model of RNA processing in human mitochondria, Nature. 290 (1981) 470-474. doi:10.1038/290470a0.

[41] D.A. Clayton, Replication of animal mitochondrial DNA, Cell. 28 (1982) 693705. doi:10.1016/0092-8674(82)90049-6.

[42] O. Rackham, J.D. Busch, S. Matic, S.J. Siira, I. Kuznetsova, I. Atanassov, et al., Hierarchical RNA Processing Is Required for Mitochondrial Ribosome Assembly, Cell Rep. (2016) 1-18. doi:10.1016/j.celrep.2016.07.031.

[43] L.K. Brzezniak, M. Bijata, R.J. Szczesny, P.P. Stepien, Involvement of human ELAC2 gene product in 3' end processing of mitochondrial tRNAs, RNA Biol. 8 (2014) 616-626. doi:10.4161/rna.8.4.15393.

[44] H. Antonicka, E.A. Shoubridge, Mitochondrial RNA Granules Are Centers for Posttranscriptional RNA Processing and Ribosome Biogenesis, Cell Rep. 10 (2015) 920-932. doi:10.1016/j.celrep.2015.01.030.

[45] E. Boehm, S. Zaganelli, K. Maundrell, A.A. Jourdain, S. Thore, J.-C. Martinou, FASTKD1 and FASTKD4 have opposite effects on expression of specific mitochondrial RNAs, depending upon their endonuclease-like RAP domain, Nucleic Acids Res. 45 (2017) 6135-6146. doi:10.1093/nar/gkx164.

[46] M. Lagouge, A. Mourier, H.J. Lee, H. Spåhr, T. Wai, C. Kukat, et al., SLIRP Regulates the Rate of Mitochondrial Protein Synthesis and Protects LRPPRC 
from Degradation, PLoS Genet. 11 (2015) e1005423-19.

doi:10.1371/journal.pgen.1005423.

[47] M.D. Metodiev, H. Spåhr, P. Loguercio Polosa, C. Meharg, C. Becker, J. Altmueller, et al., NSUN4 Is a Dual Function Mitochondrial Protein Required for Both Methylation of 12S rRNA and Coordination of Mitoribosomal Assembly, PLoS Genet. 10 (2014) e1004110-11.

doi:10.1371/journal.pgen.1004110.

[48] T.R. Richman, O. Rackham, A. Filipovska, Mitochondria: Unusual features of the mammalian mitoribosome, Int. J. Biochem. Cell Biol. 53 (2014) 115-120. doi:10.1016/j.biocel.2014.05.011.

[49] A. Amunts, A. Brown, J. Toots, S.H.W. Scheres, V. Ramakrishnan, The structure of the human mitochondrial ribosome, Sci. 348 (2015) 95-98. doi:10.1126/science.aaa1193.

[50] B.J. Greber, P. Bieri, M. Leibundgut, A. Leitner, R. Aebersold, D. Boehringer, et al., The complete structure of the 55S mammalian mitochondrial ribosome, Sci. 348 (2015) 303-308. doi:10.1126/science.aaa3872.

[51] Y.-T. Tu, A. Barrientos, The Human Mitochondrial DEAD-Box Protein DDX28 Resides in RNA Granules and Functions in Mitoribosome Assembly, Cell Rep. 10 (2015) 854-864. doi:10.1016/j.celrep.2015.01.033.

[52] T.R. Mercer, S. Neph, M.E. Dinger, J. Crawford, M.A. Smith, A.-M.J. Shearwood, et al., The Human Mitochondrial Transcriptome, Cell. 146 (2011) 645-658. doi:10.1016/j.cell.2011.06.051.

[53] J. Harmel, B. Ruzzenente, M. Terzioglu, H. Spahr, M. Falkenberg, N.G. Larsson, The Leucine-rich Pentatricopeptide Repeat-containing Protein (LRPPRC) Does Not Activate Transcription in Mammalian Mitochondria, J. Biol. Chem. 288 (2013) 15510-15519. doi:10.1074/jbc.M113.471649.

[54] I. Kühl, M. Miranda, V. Posse, D. Milenkovic, A. Mourier, S.J. Siira, et al., POLRMT regulates the switch between replication primer formation and gene expression of mammalian mtDNA, Sci. Adv. 2 (2016) e1600963-e1600963. doi:10.1126/sciadv.1600963.

[55] J. Byrnes, K. Hauser, L. Norona, E. Mejia, C. Simmerling, M. Garcia-Diaz, Base Flipping by MTERF1 Can Accommodate Multiple Conformations and Occurs in a Stepwise Fashion, J. Mol. Biol. 428 (2016) 2542-2556. doi:10.1016/j.jmb.2015.10.021.

[56] A.K. Hyvarinen, J.L.O. Pohjoismaki, A. Reyes, S. Wanrooij, T. Yasukawa, P.J. Karhunen, et al., The mitochondrial transcription termination factor mTERF modulates replication pausing in human mitochondrial DNA, Nucleic Acids Res. 35 (2007) 6458-6474. doi:10.1093/nar/gkm676.

[57] Y. Shi, V. Posse, X. Zhu, A.K. Hyvarinen, H.T. Jacobs, M. Falkenberg, et al., Mitochondrial transcription termination factor 1 directs polar replication fork pausing, Nucleic Acids Res. 44 (2016) 5732-5742. doi:10.1093/nar/gkw302.

[58] J.F. Hess, M.A. Parisi, J.L. Bennett, D.A. Clayton, Impairment of mitochondrial transcription termination by a point mutation associated with the MELAS subgroup of mitochondrial encephalomyopathies, Nature. 351 (1991) 236-239. doi: $10.1038 / 351236 \mathrm{a} 0$.

[59] C. Viscomi, A. Spinazzola, M. Maggioni, E. Fernandez-Vizarra, V. Massa, C. Pagano, et al., Early-onset liver mtDNA depletion and late-onset proteinuric nephropathy in Mpv17 knockout mice, Hum. Mol. Gen. 18 (2008) 12-26. doi:10.1093/hmg/ddn309.

[60] M. Roberti, F. Bruni, P. Loguercio Polosa, C. Manzari, M.N. Gadaleta, P. 
Cantatore, MTERF3, the most conserved member of the mTERF-family, is a modular factor involved in mitochondrial protein synthesis, Biochim. Biophys. Acta. Bioenerg. 1757 (2006) 1199-1206. doi:10.1016/j.bbabio.2006.04.026.

[61] D.C. Andersson, J. Fauconnier, C.B. Park, S.-J. Zhang, J. Thireau, N. Ivarsson, et al., Enhanced Cardiomyocyte Ca 2+Cycling Precedes Terminal AV-Block in Mitochondrial Cardiomyopathy Mterf3KO Mice, Antioxid. Redox Signal. 15 (2011) 2455-2464. doi:10.1089/ars.2011.3915.

[62] B.S. Masters, L.L. Stohl, D.A. Clayton, Yeast mitochondrial RNA polymerase is homologous to those encoded by bacteriophages T3 and T7, Cell. 51 (1987) 8999. doi:10.1016/0092-8674(87)90013-4.

[63] M. Falkenberg, N.-G. Larsson, C.M. Gustafsson, DNA Replication and Transcription in Mammalian Mitochondria, Annu. Rev. Biochem. 76 (2007) 679-699. doi:10.1146/annurev.biochem.76.060305.152028.

[64] M. Falkenberg, M. Gaspari, A. Rantanen, A. Trifunovic, N.-G. Larsson, C.M. Gustafsson, Mitochondrial transcription factors B1 and B2 activate transcription of human mtDNA, Nat. Genet. 31 (2002) 289-294. doi:10.1038/ng909.

[65] Y. Shi, A. Dierckx, P.H. Wanrooij, S. Wanrooiji, N.-G. Larsson, L.M. Wilhelmsson, et al., Mammalian transcription factor A is a core component of the mitochondrial transcription machinery, PNAS. 109 (2012) 16510-16515. doi:10.1073/pnas.1119738109/-/DCSupplemental.

[66] I. Kühl, C. Kukat, B. Ruzzenente, D. Milenkovic, A. Mourier, M. Miranda, et al., POLRMT does not transcribe nuclear genes, Nature. 514 (2014) E7-E11. doi:10.1038/nature13690.

[67] V. Posse, E. Hoberg, A. Dierckx, S. Shahzad, C. Koolmeister, N.-G. Larsson, et al., The amino terminal extension of mammalian mitochondrial RNA polymerase ensures promoter specific transcription initiation, Nucleic Acids Res. 42 (2014) 3638-3647. doi:10.1093/nar/gkt1397.

[68] E. Yakubovskaya, K.E. Guja, E.T. Eng, W.S. Choi, E. Mejia, D. Beglov, et al., Organization of the human mitochondrial transcription initiation complex, Nucleic Acids Res. 42 (2014) 4100-4112. doi:10.1093/nar/gkt1360.

[69] A. Ramachandran, U. Basu, S. Shemaila, D. Nandakumar, S.S. Patel, Human mitochondrial transcription factors TFAM and TFB2M work synergistically in promoter melting during transcription initiation, Nucleic Acids Res. (2016) 114. doi:10.1093/nar/gkw1157.

[70] M. Minczuk, J. He, A.M. Duch, T.J. Ettema, A. Chlebowski, K. Dzionek, et al., TEFM (c17orf42) is necessary for transcription of human mtDNA, Nucleic Acids Res. 39 (2011) 4284-4299. doi:10.1093/nar/gkq1224.

[71] V. Posse, S. Shahzad, M. Falkenberg, B.M. Hällberg, C.M. Gustafsson, TEFM is a potent stimulator of mitochondrial transcription elongation in vitro, Nucleic Acids Res. 43 (2015) 2615-2624. doi:10.1093/nar/gkv105.

[72] K. Agaronyan, Y.I. Morozov, M. Anikin, D. Temiakov, Replication-transcription switch in human mitochondria, Sci. 347 (2015) 548-551.

doi:10.1126/science.aaa0986.

[73] Y.V. Surovtseva, T.E. Shutt, J. Cotney, H. Cimen, S.Y. Chen, E.C. Koc, et al., Mitochondrial Ribosomal Protein L12 selectively associates with human mitochondrial RNA polymerase to activate transcription, PNAS. 108 (2011) 17921-17926. doi:10.1073/pnas.1108852108/-/DCSupplemental.

[74] Z. Wang, J. Cotney, G.S. Shadel, Human Mitochondrial Ribosomal Protein MRPL12 Interacts Directly with Mitochondrial RNA Polymerase to Modulate Mitochondrial Gene Expression, J. Biol. Chem. 282 (2007) 12610-12618. 
doi:10.1074/jbc.M700461200.

[75] J. Nouws, A.V. Goswami, M. Bestwick, B.J. McCann, Y.V. Surovtseva, G.S. Shadel, Mitochondrial Ribosomal Protein L12 Is Required for POLRMT Stability and Exists as Two Forms Generated by Alternative Proteolysis during Import, J. Biol. Chem. 291 (2016) 989-997. doi:10.1074/jbc.M115.689299.

[76] Y.V. Surovtseva, G.S. Shadel, Transcription-independent role for human mitochondrial RNA polymerase in mitochondrial ribosome biogenesis, Nucleic Acids Res. 41 (2013) 2479-2488. doi:10.1093/nar/gks1447.

[77] M.D. Metodiev, N. Lesko, C.B. Park, Y. Cámara, Y. Shi, R. Wibom, et al., Methylation of 12S rRNA Is Necessary for In Vivo Stability of the Small Subunit of the Mammalian Mitochondrial Ribosome, Cell Metab. 9 (2009) 386397. doi:10.1016/j.cmet.2009.03.001.

[78] K. Schwinghammer, A.C.M. Cheung, Y.I. Morozov, K. Agaronyan, D. Temiakov, P. Cramer, Structure of human mitochondrial RNA polymerase elongation complex, Nat. Struct. Mol. Biol. 20 (2013) 1298-1303. doi:10.1038/nsmb.2683.

[79] E. Vilardo, C. Nachbagauer, A. Buzet, A. Taschner, J. Holzmann, W. Rossmanith, A subcomplex of human mitochondrial RNase $\mathrm{P}$ is a bifunctional methyltransferase--extensive moonlighting in mitochondrial tRNA biogenesis, Nucleic Acids Res. 40 (2012) 11583-11593. doi:10.1093/nar/gks910.

[80] G. Liu, T.R. Mercer, A.-M.J. Shearwood, S.J. Siira, M.E. Hibbs, J.S. Mattick, et al., Mapping of Mitochondrial RNA-Protein Interactions by Digital RNase Footprinting, Cell Rep. 5 (2013) 839-848. doi:10.1016/j.celrep.2013.09.036.

[81] S.-Y. Yang, X.-Y. He, H. Schulz, Multiple functions of type $1017 \beta-$ hydroxysteroid dehydrogenase, Trends Endocrinol. Metab. 16 (2005) 167-175. doi:10.1016/j.tem.2005.03.006.

[82] E. Vilardo, W. Rossmanith, Molecular insights into HSD10 disease: impact of SDR5C1 mutations on the human mitochondrial RNase P complex, Nucleic Acids Res. 43 (2015) 5112-5119. doi:10.1093/nar/gkv408.

[83] J. Zschocke, HSD10 disease: clinical consequences of mutations in the HSD17B10 gene, J. Inherit. Metab. Dis. 35 (2011) 81-89. doi:10.1007/s10545011-9415-4.

[84] J. Holzmann, W. Rossmanith, tRNA recognition, processing, and disease: Hypotheses around an unorthodox type of RNase P in human mitochondria, Mitochondrion. 9 (2009) 284-288. doi:10.1016/j.mito.2009.03.008.

[85] W. Rossmanith, J. Holzmann, Processing mitochondrial (t)RNAs: New enzyme, old job, Cell Cycle. 8 (2014) 1650-1653. doi:10.4161/cc.8.11.8502.

[86] M. Lechner, W. Rossmanith, R.K. Hartmann, C. Thölken, B. Gutmann, P. Giegé, et al., Distribution of Ribonucleoprotein and Protein-Only RNase P in Eukarya, Mol. Biol. Evol. (2015) msv187-8. doi:10.1093/molbev/msv187.

[87] A. Gobert, B. Gutmann, A. Taschner, M. Gößringer, J. Holzmann, R.K. Hartmann, et al., A single Arabidopsis organellar protein has RNase P activity, Nat. Struct. Mol. Biol. 17 (2010) 740-744. doi:10.1038/nsmb.1812.

[88] A. Taschner, C. Weber, A. Buzet, R.K. Hartmann, A. Hartig, W. Rossmanith, Nuclear RNase P of Trypanosoma brucei: A Single Protein in Place of the Multicomponent RNA-Protein Complex, Cell Rep. 2 (2012) 19-25. doi:10.1016/j.celrep.2012.05.021.

[89] G. Bonnard, A. Gobert, M. Arrive, F. Pinker, T. Salinas-Giege, P. Giegé, Transfer RNA maturation in Chlamydomonas mitochondria, chloroplast and the nucleus by a single RNase P protein, Plant J. 87 (2016) 270-280. 
doi:10.1111/tpj.13198.

[90] M. Howard, W.H. Lim, C. Fierke, M. Koutmos, Mitochondrial ribonuclease P structure provides insight into the evolution of catalytic strategies for precursortRNA 5' processing, PNAS. 109 (2012) 1-6. doi:10.1073/pnas.1209062109//DCSupplemental/pnas.201209062SI.pdf.

[91] A.A. Jourdain, M. Koppen, M. Wydro, C.D. Rodley, R.N. Lightowlers, Z.M. Chrzanowska-Lightowlers, et al., GRSF1 Regulates RNA Processing in Mitochondrial RNA Granules, Cell Metab. 17 (2013) 399-410. doi:10.1016/j.cmet.2013.02.005.

[92] H. Antonicka, F. Sasarman, T. Nishimura, V. Paupe, E.A. Shoubridge, The Mitochondrial RNA-Binding Protein GRSF1 Localizes to RNA Granules and Is Required for Posttranscriptional Mitochondrial Gene Expression, Cell Metab. 17 (2013) 386-398. doi:10.1016/j.cmet.2013.02.006.

[93] A.A. Jourdain, E. Boehm, K. Maundrell, J.-C. Martinou, Mitochondrial RNA granules: Compartmentalizing mitochondrial gene expression, J. Cell. Biol. 212 (2016) 611-614. doi:10.1083/jcb.201507125.

[94] M.I.G. Lopez Sanchez, A.-M.J. Shearwood, T. Chia, S.M.K. Davies, O. Rackham, A. Filipovska, Estrogen-Mediated Regulation of Mitochondrial Gene Expression, Mol. Endocrinol. 29 (2015) 14-27. doi:10.1210/me.2014-1077.

[95] I. Kuznetsova, S.J. Siira, A.-M.J. Shearwood, J.A. Ermer, A. Filipovska, O. Rackham, Simultaneous processing and degradation of mitochondrial RNAs revealed by circularized RNA sequencing, Nucleic Acids Res. (2017) 1-14. doi:10.1093/nar/gkx104.

[96] O. Rackham, A. Filipovska, The role of mammalian PPR domain proteins in the regulation of mitochondrial gene expression, Biochim. Biophys. Acta. 1819 (2012) 1008-1016. doi:10.1016/j.bbagrm.2011.10.007.

[97] H. Spåhr, A. Rozanska, X. Li, I. Atanassov, R.N. Lightowlers, Z.M.A. Chrzanowska-Lightowlers, et al., SLIRP stabilizes LRPPRC via an RRM-PPR protein interface, Nucleic Acids Res. (2016) gkw575-15. doi:10.1093/nar/gkw575.

[98] F. Xu, J.B.L. Addis, J.M. Cameron, B.H. Robinson, LRPPRC mutation suppresses cytochrome oxidase activity by altering mitochondrial RNA transcript stability in a mouse model, Biochem. J. 441 (2012) 275-283. doi:10.1042/BJ20110985.

[99] C. Morin, G.A. Mitchell, J. Larochelle, M. Lambert, H. Ogier, B.H. Robinson, et al., Clinical, Metabolic, and Genetic Aspects of Cytochrome C Oxidase Deficiency in Saguenay-Lac-Saint-Jean, Am. J. Hum. Genet. 53 (1993) 488-496.

[100] F. Merante, R. Petrova-Benedict, N. MacKay, G. Mitchell, M. Lambert, C. Morin, et al., A biochemically distinct form of cytochrome oxidase (COX) deficiency in the Saguenay-Lac-Saint-Jean region of Quebec, Am. J. Hum. Genet. 53 (1993) 481-487.

[101] F.G. Debray, C. Morin, A. Janvier, J. Villeneuve, B. Maranda, R. Laframboise, et al., LRPPRC mutations cause a phenotypically distinct form of Leigh syndrome with cytochrome c oxidase deficiency, J. Med, Genet. 48 (2011) 183189. doi:10.1136/jmg.2010.081976.

[102] M. Oláhová, S.A. Hardy, J. Hall, J.W. Yarham, T.B. Haack, W.C. Wilson, et al., LRPPRC mutations cause early-onset multisystem mitochondrial disease outside of the French-Canadian population, Brain. 138 (2015) 3503-3519. doi:10.1093/brain/awv291.

[103] F. Sasarman, T. Nishimura, H. Antonicka, W. Weraarpachai, E.A. Shoubridge, 
LSFC Consortium, et al., Tissue-specific responses to the LRPPRC founder mutation in French Canadian Leigh Syndrome, Hum. Mol. Gen. 24 (2014) 480491. doi:10.1093/hmg/ddu468.

[104] A. Mourier, B. Ruzzenente, T. Brandt, W. Kühlbrandt, N.-G. Larsson, Loss of LRPPRC causes ATP synthase deficiency, Hum. Mol. Genet. 23 (2014) 25802592. doi:10.1093/hmg/ddt652.

[105] A. Cuillerier, V. Cadete, S. Deschênes, Y. Burelle, L. Consortium, Histopathology and Mitochondrial Function in Liver-Specific LRPPRC Knockout Mice, FASEB J. 29 (2015).

[106] A.R. Wolf, V.K. Mootha, Functional Genomic Analysis of Human Mitochondrial RNA Processing, Cell Rep. 7 (2014) 918-931. doi:10.1016/j.celrep.2014.03.035.

[107] S. Siira, H. Spåhr, A.-M.J. Shearwood, B. Ruzzenente, N.-G. Larsson, et al., LRPPRC-mediated folding of the mitochondrial transcriptome, Nature Comm. (2017) NCOMMS-17-09377B.

[108] T. Chujo, T. Ohira, Y. Sakaguchi, N. Goshima, N. Nomura, A. Nagao, et al., LRPPRC/SLIRP suppresses PNPase-mediated mRNA decay and promotes polyadenylation in human mitochondria, Nucleic Acids Res. 40 (2012) 80338047. doi:10.1093/nar/gks506.

[109] W.C. Wilson, H.-T. Hornig-Do, F. Bruni, J.H. Chang, A.A. Jourdain, J.-C. Martinou, et al., A human mitochondrial poly(A) polymerase mutation reveals the complexities of post-transcriptional mitochondrial gene expression, Hum. Mol. Genet. 23 (2014) 6345-6355. doi:10.1093/hmg/ddu352.

[110] K.L. Perks, N. Ferreira, T.R. Richman, J.A. Ermer, I. Kuznetsova, A.-M.J. Shearwood, et al., Adult-onset obesity is triggered by impaired mitochondrial gene expression, Sci. Adv. (2017) 3, e1700677.

[111] O. Rackham, A.-M.J. Shearwood, T.R. Mercer, S.M.K. Davies, J.S. Mattick, A. Filipovska, Long noncoding RNAs are generated from the mitochondrial genome and regulated by nuclear-encoded proteins, RNA. 17 (2011) 2085-2093. doi:10.1261/rna.029405.111.

[112] E.C. Koc, H. Cimen, B. Kumcuoglu, N. Abu, G. Akpinar, M.E. Haque, et al., Identification and characterization of CHCHD1, AURKAIP1, and CRIF1 as new members of the mammalian mitochondrial ribosome, Front. Physiol. 4 (2013) 115. doi:10.3389/fphys.2013.00183.

[113] E.C. Koc, L.L. Spremulli, Identification of Mammalian Mitochondrial Translational Initiation Factor 3 and Examination of Its Role in Initiation Complex Formation with Natural mRNAs, J. Biol. Chem. 277 (2002) 3554135549. doi:10.1074/jbc.M202498200.

[114] R. Richter, J. Rorbach, A. Pajak, P.M. Smith, H.J. Wessels, M.A. Huynen, et al., A functional peptidyl-tRNA hydrolase, ICT1, has been recruited into the human mitochondrial ribosome, EMBO J. 29 (2010) 1116-1125. doi:10.1038/emboj.2010.14.

[115] M.E. Haque, H. Koc, H. Cimen, E.C. Koc, L.L. Spremulli, Contacts between mammalian mitochondrial translational initiation factor 3 and ribosomal proteins in the small subunit, Biochim. Biophys. Acta - Proteins Proteomics. 1814 (2011) 1779-1784. doi:10.1016/j.bbapap.2011.09.013.

[116] S. Akabane, T. Ueda, K.H. Nierhaus, N. Takeuchi, Ribosome Rescue and Translation Termination at Non-Standard Stop Codons by ICT1 in Mammalian Mitochondria, PLoS Genet. 10 (2014) e1004616-14.

doi:10.1371/journal.pgen.1004616. 
[117] P.S. Kaushal, M.R. Sharma, T.M. Booth, E.M. Haque, C.S. Tung, K.Y. Sanbonmatsu, et al., Cryo-EM structure of the small subunit of the mammalian mitochondrial ribosome, Proc. Natl. Acad. Sci. USA. 111 (2014) 7284-7289. doi:10.1073/pnas.1401657111.

[118] P. Smits, J.A.M. Smeitink, L.P. van den Heuvel, M.A. Huynen, T.J.G. Ettema, Reconstructing the evolution of the mitochondrial ribosomal proteome, Nucleic Acids Res. 35 (2007) 4686-4703. doi:10.1093/nar/gkm441.

[119] E.C. Koc, A. Ranasinghe, W. Burkhart, K. Blackburn, H. Koc, A. Moseley, et al., A new face on apoptosis: death-associated protein 3 and PDCD9 are mitochondrial ribosomal proteins, FEBS Lett. 492 (2001) 166-170. doi:10.1016/S0014-5793(01)02250-5.

[120] S. Dennerlein, A. Rozanska, M. Wydro, Z.M.A. Chrzanowska-Lightowlers, R.N. Lightowlers, Human ERAL1 is a mitochondrial RNA chaperone involved in the assembly of the $28 \mathrm{~S}$ small mitochondrial ribosomal subunit, Biochem. J. 430 (2010) 551-558. doi:10.1042/BJ20100757.

[121] T. Tang, B. Zheng, S.H. Chen, A.N. Murphy, K. Kudlicka, Z. Huilin, et al., hNOA1 Interacts with Complex I and DAP3 and Regulates Mitochondrial Respiration and Apoptosis, J. Biol. Chem. 284 (2009) 5414-5424. doi:0.1074/jbc.M807797200.

[122] M.R. Karpenahalli, A.N. Lupas, J. Söding, TPRpred: a tool for prediction of TPR-, PPR- and SEL-I-like repeats from protein sequences, BMC Bioinformatics. 8 (2007) 2-8. doi:10.1186/1471-2105-8-2. 
Figure 1

\begin{tabular}{|c|c|c|}
\hline MTERF1 & 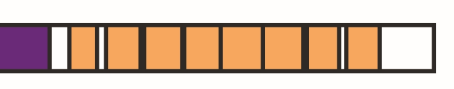 & $\square$ PPRs \\
\hline MTERF2 & 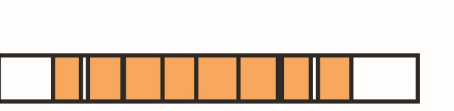 & $\square$ MTERF repeats \\
\hline MTERF3 & \|\|$\square\|\square\| \|$ & - 50 aа \\
\hline
\end{tabular}

MTERF4 $\square[D \mid D \| \amalg$

POLRMT $\begin{gathered}\text { N-terminal } \\ \text { extension }\end{gathered}$

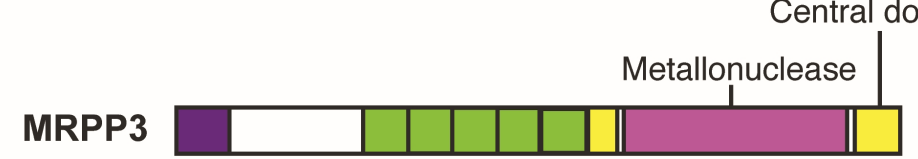

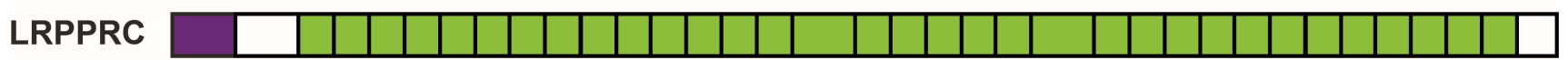

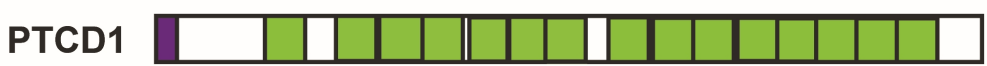

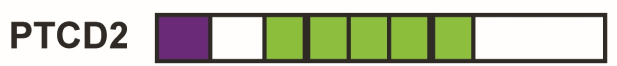

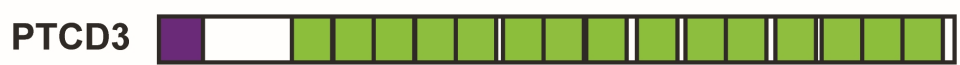

MRPS27 
Figure 2
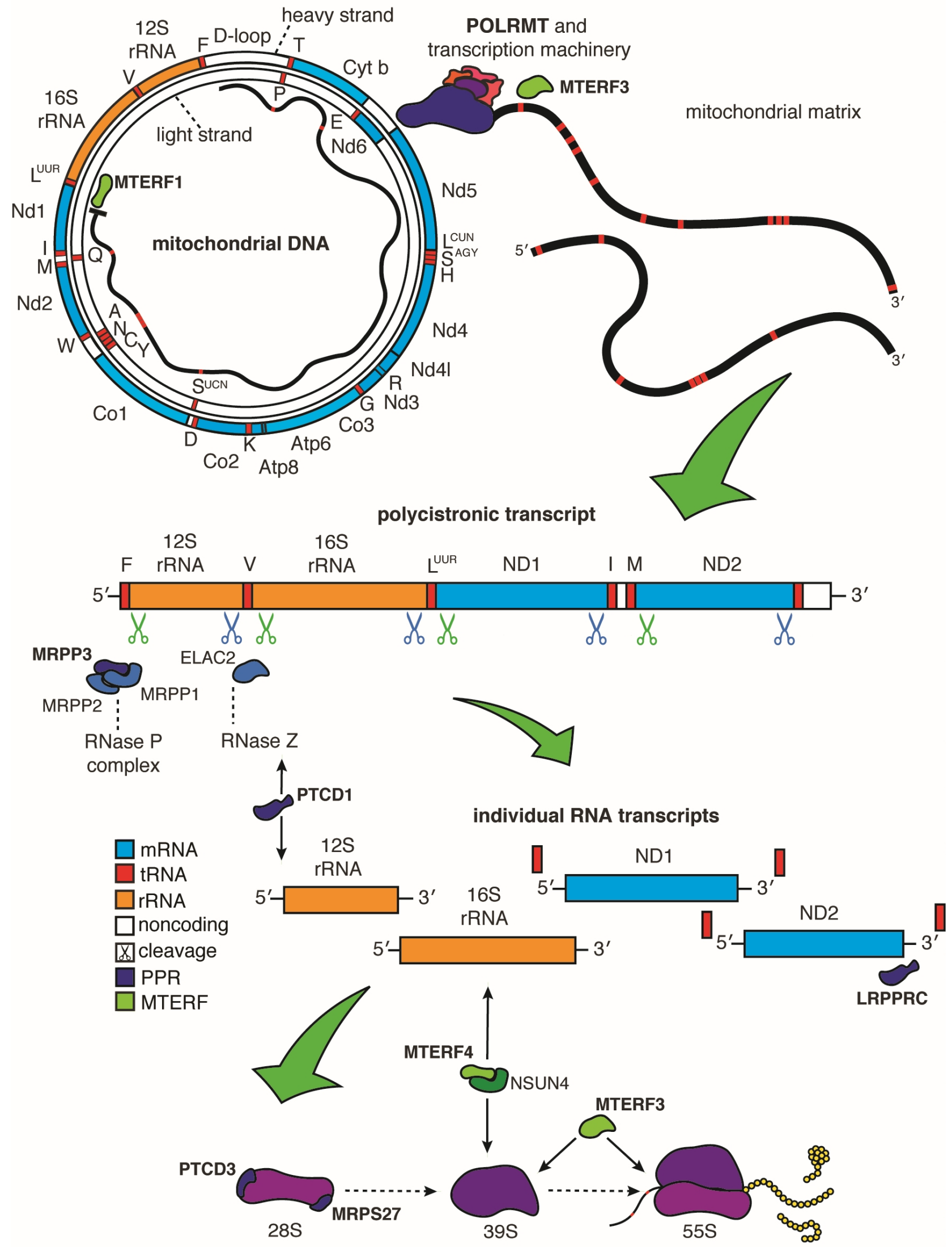
Figure 3

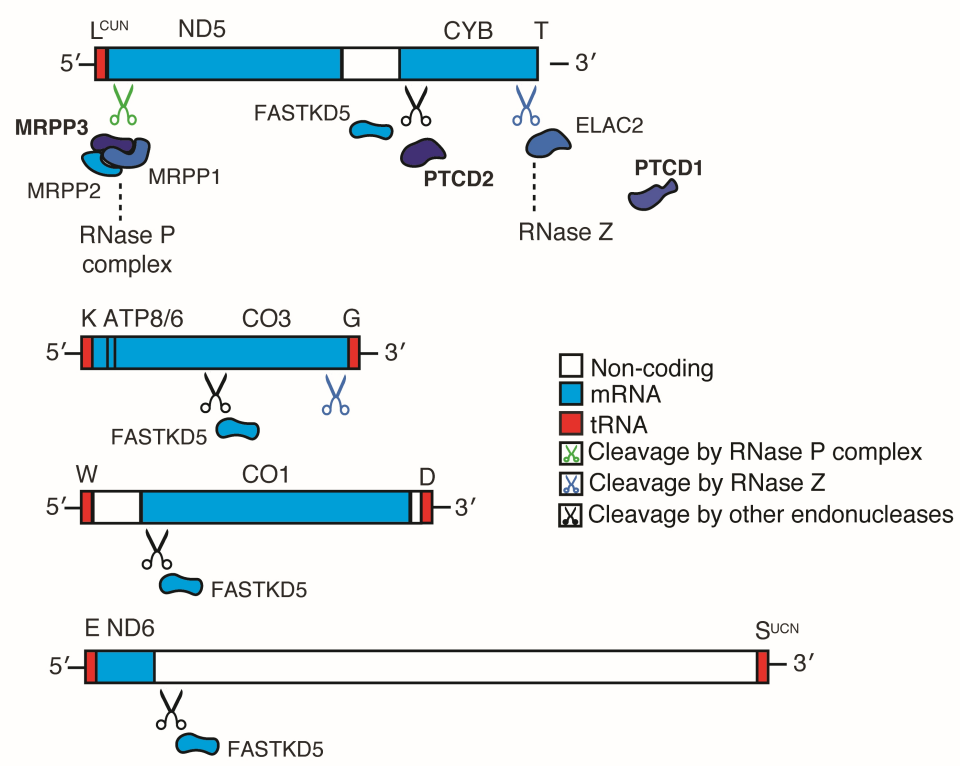

